\title{
Anxiety, Stress, Depression, and Patients' Responses to Periodontal Treatment: Periodontists' Knowledge and Professional Behavior
}

\author{
Paul W. Kloostra, ${ }^{*}$ Robert M. Eber, ${ }^{\dagger}$ and Marita Rohr Inglehart ${ }^{\dagger \dagger}$
}

Background: Anxiety, stress, and depression affect the use of health care services, treatment decision-making, and responses to periodontal treatment. This study explored periodontists' confidence in detecting patient anxiety, stress, or depression, as well as their knowledge concerning the relationships between these factors and patients' pain, use of pain medication, and wound healing after periodontal treatment. In addition, this research surveyed if (and which) special accommodations were offered when treating patients with high levels of anxiety, stress, or depression.

Methods: Data were collected from 171 members of the American Academy of Periodontology (response rate $=34.41 \%$ ). Most respondents were male $(82.2 \%)$, white $(88.2 \%)$, and practiced in solo practices $(60.9 \%)$.

Results: The respondents were more knowledgeable about the effects of anxiety and stress on pain, the use of pain medication, and wound healing than about the impact of depression on these outcomes. They agreed more strongly with statements that they were more confident in their ability to perceive when patients were anxious and stressed than when they were depressed. They also offered more special accommodations for patients with anxiety and stress than for patients with depression.

Conclusions: The respondents were significantly less knowledgeable about the impact of depression on patients' responses to periodontal treatment than about the effect of anxiety and stress. Given the evidence concerning the relationships among depression, pain, pain medication use, and wound healing, it is important to educate periodontists about the role of anxiety and stress and the significance of depression on their patients' responses to periodontal therapy. J Periodontol 2007;78:64-71.

\section{KEY WORDS}

Anxiety; depression; pain; stress; treatment; wound healing.

\footnotetext{
* School of Dentistry, University of Michigan, Ann Arbor, MI.

$\uparrow$ Department of Periodontics and Oral Medicine, School of Dentistry, University of Michigan.

$\ddagger$ Department of Psychology, College of Literature, Science and Arts, University of Michigan.
}

In n 1995, the Institute of Medicine ${ }^{1}$ published a report about the future of dental education. One of the strongest themes developed in this report was the call to educate more patient-centered providers. One question is whether this recommendation is as applicable to the field of periodontology as it is to general dentistry. Most periodontists might agree that patient-provider communication is a key factor for a successful practice. However, it also is important to explore whether surgically oriented practitioners understand the significance that psychosocial factors have on their patients' responses to periodontal treatment. Therefore, the objectives of this research were to explore whether periodontists 1) are knowledgeable about how three psychosocial factors (i.e., anxiety, depression, and stress) affect their patients' responses to periodontal treatment, 2) are confident in their ability to recognize these psychological states in their patients, and 3) are willing to consider the significance of these factors in their professional practice by offering special accommodations for patients who are anxious, depressed, or stressed.

Anxiety and affective disorders, especially depression, are among the most prevalent mental disorders in adults in the United States. In addition, $\sim 35 \%$ of the United States population $>18$ years of age experiences adverse health effects 
that are due to stress. ${ }^{2}$ Depression ranks in the top 10 causes of disability worldwide. ${ }^{3}$ In a study covering a 40-year span, Murphy et al. ${ }^{4}$ showed that chronic depression has maintained a consistent prevalence of $\sim 5 \%$ since 1970. Depression has been associated with anxiety. ${ }^{5}$ According to the Surgeon General's Report on Mental Health, ${ }^{2}$ depression and anxiety disorders affect twice as many women as men.

\section{The Role of Anxiety in Dentistry}

The role of anxiety in dentistry is complex and powerful, as described convincingly in the monograph by Milgrom et al. ${ }^{6}$ Dental anxiety/fear is quite prevalent among children and adults; it prevents patients from seeking dental care and affects their treatment decision making. ${ }^{7}$ Research showed that dental anxiety causes patients to cancel, miss, or arrive late for dental appointments. ${ }^{6}$ Research investigating patient responses to surgery showed that patients with increased preoperative anxiety and greater expectations of pain experienced greater postoperative pain. ${ }^{8}$ Patients who were given psychological interventions aimed at reducing anxiety/stress reported significantly less postoperative pain when undergoing periodontal surgery compared to patients who did not receive these interventions. ${ }^{9}$ Research also showed that patients who learned about non-pharmacological strategies to manage pain used less pain medication. ${ }^{10}$ In general, there is a close relationship between anxiety and pain; anxiety even may be considered a predictor of pain. ${ }^{11,12}$

Research also showed that there is empirical support for the hypothesis that anxiety affects wound healing. For example, patients using relaxationguided imagery exhibited less anxiety and less wound inflammation following surgical procedures than patients not using these techniques. ${ }^{13}$ George et al. ${ }^{14}$ and George and Scott ${ }^{15}$ demonstrated that giving surgery a more positive meaning improved patients' acceptance of their condition, made their expectations more positive, and reduced their anxiety about recovery; these resulted in improved wound healing. Recent research by Johannsen et al. ${ }^{16}$ showed that higher levels of anxiety impaired wound healing and were associated with an increase in the severity of periodontal disease.

\section{The Role of Stress in Dentistry}

Recent research also demonstrated that stress plays an important role in predicting the severity of pain, periodontal disease, ${ }^{17,18}$ and wound healing. ${ }^{19-21}$ For example, Montgomery and Bovbjerg ${ }^{19}$ showed that presurgical distress contributed to patients' postsurgical outcomes, including pain intensity. Periodontal disease was affected by stress and patients' ability to use effective coping methods. ${ }^{20,21}$ In addition, there is growing evidence that stress affects wound healing. According to a study by Rozlog et al., ${ }^{22}$ patients experiencing stress were slower in their recovery from periodontal surgery compared to patients not experiencing stress. In addition, healing following periodontal surgery was impacted negatively by patients' inability to use effective coping mechanisms. ${ }^{23}$ In 2004, Takada et al. ${ }^{24}$ showed that increased stress modulated the progression of periodontal inflammation. This probably resulted from an increase in interleukin-1, -4 , and -8 , which, in turn, resulted in greater periodontal destruction. ${ }^{25}$ The research on the relationship between stress and wound healing covers a wide spectrum of studies, from research conducted by Kiecolt-Glaser et al. ${ }^{26}$ in 1995 on the effect of stress on reduced cellular immune responses and, as a consequence, delayed healing of punch biopsies to extensive research findings on the relationship between chronic stressors (e.g., caring for a debilitated relative) and delayed wound healing. ${ }^{27}$

\section{The Role of Depression in Dentistry}

In addition to the research on the relationship between anxiety and stress with pain and wound healing, research documented that there is a relationship between depression and oral health ${ }^{28-30}$ and between depression and pain and wound healing. Studies by Eremenko et al. ${ }^{31}$ and by Arpino et al. ${ }^{32}$ showed that depression was correlated directly with postoperative pain and the amount of analgesics needed to alleviate pain. Patients whose depression was treated with antidepressants reported a decreased severity of postoperative pain compared to depressed patients without medication. ${ }^{32}$ Field et al. ${ }^{33}$ found that patients receiving therapeutic massages prior to burn debridement reported less pain, decreased depression, and better long-term outcomes. Eggen ${ }^{34}$ showed that depressed patients were likely to report more pain and to use more pain medication than non-depressed patients.

Research showed that depression is related to the amount of pain patients reported 31,32 and the amount of medication used, ${ }^{32}$ and it also affected patients' wound healing. Cole-King and Harding ${ }^{35}$ established that depressed patients displayed delayed wound healing compared to non-depressed patients. In addition, Elter et al. ${ }^{36}$ found that clinical depression had a negative effect on periodontal treatment outcome 1 year after treatment. In a study exploring factors affecting patients with different types of periodontitis (rapidly progressive periodontitis, chronic periodontitis, and no significant periodontal destruction), depression was a significant factor in distinguishing among patients in these three groups. ${ }^{37}$

In summary, given the fact that anxiety, stress, and depression are quite prevalent among adults in the United States and that these psychosocial conditions clearly affect patients' pain perception, their use of pain medication, and wound healing, it seems important 
to explore whether periodontists 1) are knowledgeable about the relationships between these psychosocial factors and patients' responses to treatment, 2 ) are confident in their ability to identify anxious, stressed, or depressed patients, and 3) modify their interactions and treatment for these patients by offering special accommodations.

\section{MATERIALS AND METHODS}

This research was approved by the Institutional Review Board for the Health Sciences at the University of Michigan.

\section{Respondents}

During the summer of 2005, a survey was mailed to a random sample of 500 members of the American Academy of Periodontology (AAP). Three surveys could not be delivered, and 171 members responded (141 males, 28 females, and two missing data for gender); the response rate was $34.41 \%$. The respondents ranged in age from 31 to 66 years (average age, 50.36 years; SD: 8.630 ). They were predominantly white $(88.2 \%)$ and practiced in solo practices $(60.9 \%)$. An analysis of the institutions where the respondents received their graduate training in periodontics showed that $11.7 \%$ were located in AAP district 1 (Connecticut, Maine, Massachusetts, New Hampshire, Rhode Island, and Vermont; the province of Quebec and maritime provinces of Canada); $4.1 \%$ were located in district 2 (Delaware, District of Columbia, Maryland, Pennsylvania, and West Virginia and the following cities and counties of Virginia: Alexandria, Falls Church, Fairfax City, Vienna, Arlington County, and Loudon County); 16.4\% were located in district 3 (Alabama, Florida, Georgia, Kentucky, Missouri, North Carolina, South Carolina, Tennesee, Guam, Virgin Islands, and Virginia [excluding those included in district 2]; $17 \%$ were located in district 4 (Illinois, Indiana, lowa, Kansas, Michigan, Minnesota, Missouri, North Dakota, Ohio, South Dakota, and Wisconsin; provinces of Ontario and Manitoba, Canada); $12.3 \%$ were located in district 5 (Arizona, Colorado, Louisiana, Nebraska, Oklahoma, Puerto Rico, and Texas); $11.1 \%$ were located in district 6 (Alaska, Arizona, California, Hawaii, Idaho, Montana, Nevada, New Mexico, Oregon, Utah, Washington, and Wyoming; provinces of British Columbia, Alberta, and Saskatchewan, Canada); $6.4 \%$ were located in district 7 (New Jersey and New York); and 11.7\% were located in district 8 (Federal Dental Services and employees of the Federal Government, including all branches of federal services, both civilian and military). Approximately $10 \%$ of respondents did not report the institution where they received their graduate training.

\section{Procedure}

The survey was mailed with a cover letter by the dean of the School of Dentistry, University of Michigan, that explained the purpose of the study and requested return of the survey anonymously in a self-addressed, stamped return envelope.

\section{Materials}

The survey was three pages long. The first page contained questions concerning the respondents' sociodemographic and educational background as well as their practice characteristics. The second page consisted of 24 statements with a five-point answering scale ranging from $1=$ "disagree strongly" to $5=$ "agree strongly." These statements were used to assess the respondents' knowledge concerning the impact of anxiety, stress, and depression on patients' responses to periodontal treatment; their confidence in detecting anxiety, stress, and depression in their patients; and how well their education prepared them to consider these issues. More specifically, three statements each were concerned with the relationship between these psychological states and perceived pain ("There is a relationship between anxiety [depression/stress] and perceived pain."), the use of pain medication ("Patients that have lower anxiety [depression/stress] levels will use less pain medication."), wound integrity ("Patients will present with better wound integrity if they experience less anxiety [depression/stress]."), the provider's confidence in detecting these states ("I am confident in my ability to perceive when my patients feel anxious [depressed/stressed]."), and the degree to which dental education had prepared the provider to treat patients with these states ("Dental school prepared me well for treating patients with high levels of anxiety [depression/stress].") (Fig. 1).

The last page inquired about the respondents' professional behavior when treating patients with anxiety, stress, and depression. The respondents were asked whether they used special techniques ("Are there any special techniques you use with patients that may be experiencing high levels of anxiety [depression/stress]?"), offered special scheduling arrangements, gave special instructions before the treatment, or had specific pharmacological considerations when treating these patients. Besides asking, in a yes/no format, whether the respondents engaged in these activities, open-ended follow-up questions asked for a description of the special techniques, scheduling arrangements, instructions, and pharmacological considerations that they used.

\section{Statistical Analyses}

Analyses of variance were used to compare average responses on the five-point answer scales for the questions concerned with anxiety versus depression versus stress. It was assumed that these variables were measured on an interval measurement scale. 


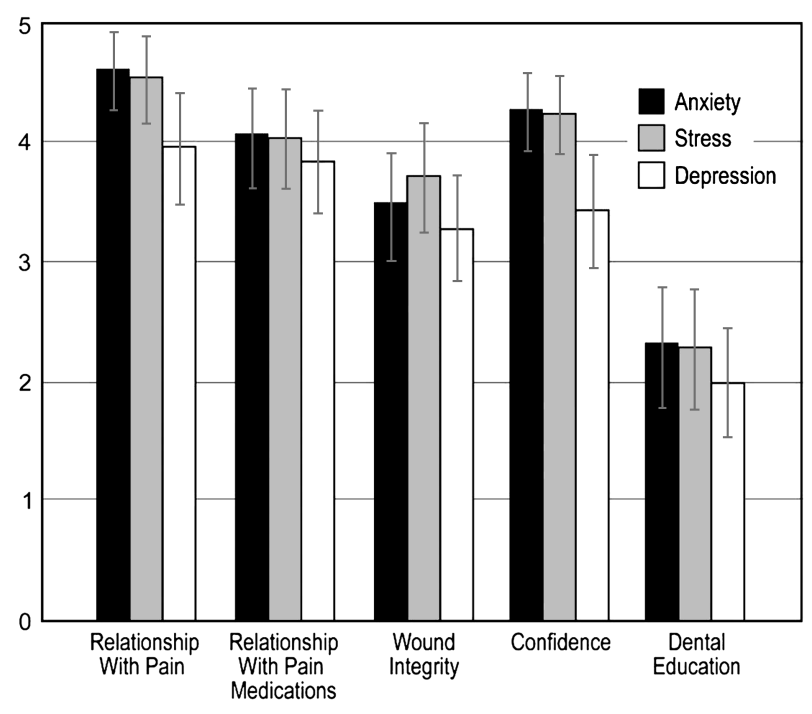

Figure I.

Periodontists' average responses concerning the role of anxiety, stress, and depression when treating periodontal patients and their educational experiences concerning the importance of psychosocial factors. The answers were given on a scale from I = "disagree strongly" to 5 = "agree strongly."

Chi-square tests were used to analyze the frequencies of responses to the questions answered with "yes" versus "no." Correlational analyses were used to assess whether there were significant relationships between periodontists' background characteristics and their responses concerning the role of psychosocial factors.

\section{RESULTS}

The first objective was to assess whether periodontists were knowledgeable concerning the degree to which anxiety, stress, and depression affect their patients' responses to periodontal treatment. As can be seen in Figure 1, the respondents agreed strongly with the statements that there is a relationship between anxiety and pain (five-point answer scale; mean $=4.64$ ) and between stress and pain (mean =4.57). However, although they agreed that there is a relationship between depression and pain (mean $=3.94)$, this average score was significantly lower than the other two means $(P<0.001)$. The same pattern of responses was found for the responses to the statements concerning the relationships between anxiety, stress, and depression and the use of pain medication (4.19 versus 4.07 versus 3.80 , respectively; $P<0.001)$ and wound integrity (3.50 versus 3.71 versus 3.32 , respectively; $P<0.001)$. For all three patient outcome variables (pain, use of pain medication, and wound integrity), respondents agreed more strongly with the statements that anxiety and stress affected these out- comes than with the statements that there was a relationship between depression and these outcomes.

The second objective was to determine whether the periodontists were confident in their abilities to recognize anxiety, stress, and depression in their patients. Their confidence level was rather high when they responded to the statements concerning detecting anxiety $($ mean $=4.31)$ and stress $($ mean $=4.29)($ Fig. 1$)$. However, confidence in their ability to identify depressed patients was significantly lower (mean $=3.45$; $P<0.001)$.

One potential reason for the differential responses might be a lack of education concerning the effects of depression on patients' responses to treatment in a dental office. The periodontists' responses to the statement, "Dental school prepared me well to treat patients with high levels of depression" was rather low (mean $=2.01$ ), which indicated that they disagreed with this statement on average. The responses concerning depression were significantly lower than the responses to the statements concerning anxiety (mean = 2.38) and stress (mean $=2.34 ; P<0.001$ ) (Fig. 1). Overall, the periodontists did not believe that their dental education had prepared them well for treating patients with high levels of anxiety, stress, and depression.

The third objective was to determine how many periodontists were willing to consider the significance of these factors in their professional practice by using special techniques when treating patients who are anxious, stressed, or depressed. Four questions assessed the respondents' professional behavior. The first question asked whether the periodontists used special techniques when treating patients with anxiety, stress, and depression. As can be seen in Table 1, 87.7\% of the respondents used special techniques when treating patients with high levels of anxiety, $78.9 \%$ used special techniques when treating patients with high levels of stress, but only $46.2 \%$ used special techniques when treating patients with high levels of depression.

Three more specific questions asked whether the providers used special scheduling arrangements, special instructions, or special pharmacological techniques when treating these three groups of patients. Significantly more providers used these techniques with anxious and stressed patients than with depressed patients. Special scheduling arrangements were made by $50.9 \%$ of providers for anxious patients, by $40.9 \%$ for patients with high stress levels, and by only $26.3 \%$ for patients with depression. More providers indicated that they gave special instructions to anxious patients (55.6\%) than to stressed patients $(41.5 \%)$ or depressed patients $(26.3 \%)$. This pattern also was found concerning pharmacological techniques used (anxious patients, $80.1 \%$; stressed patients, 60.8\%; depressed patients, 26.3\%). 
In addition to analyzing the frequencies of answers to these four questions, a sum score was computed by adding up the "yes" responses to the four questions. Table 2 shows that the providers offered, on average, 2.84 special accommodations for patients with anxiety, 2.32 special accommodations for patients with stress, but only 1.32 accommodations for patients with depression $(P<0.001)$.

A separate, open-ended follow-up question was asked for each of the three patient groups if the respondent used special techniques when treating these patients. Table 2 provides an overview of the specific techniques the providers named. The open- ended responses were organized into three categories: pharmacological behavior management, non-pharmacological management considerations, and other considerations (e.g., scheduling or referrals). When treating patients with anxiety and stress, pharmacological considerations were named most frequently, followed by non-pharmacological considerations. One hundred forty-six (85.4\%) periodontists used pharmacological management techniques when treating anxious patients, and 97 (56.7\%) used these considerations for stressed patients. However, only 29 (17.0\%) providers reported using special pharmacological techniques for patients with

Table I. Periodontists' Use of Special Techniques and/or Other Special Considerations
When Treating Patients With Anxiety, Stress, and Depression

\begin{tabular}{lcccc}
\hline N (\%) Periodontists Who... & Anxiety & Stress & Depression & $P$ \\
\hline Use special techniques & $150(87.7 \%)$ & $135(78.9 \%)$ & $79(46.2 \%)$ & $<0.001$ \\
Offer special scheduling arrangements & $87(50.9 \%)$ & $70(40.9 \%)$ & $45(26.3 \%)$ & $<0.00$ I \\
Give special instructions & $95(55.6 \%)$ & $71(41.5 \%)$ & $43(25.1 \%)$ & $<0.001$ \\
Use pharmacological techniques & $137(80.1 \%)$ & $104(60.8 \%)$ & $45(26.3 \%)$ & $<0.001$ \\
Sum of special arrangements* & 2.842 & 2.316 & 1.323 & $<0.001$ \\
\hline
\end{tabular}

* A sum score was computed by adding up the items to which the providers had responded in a positive manner. This sum score could range from 0 to 4

A univariate analysis of variance was used to compare the three average sum scores.

Table 2.

Responses Concerning Special Techniques Used When Treating Anxious, Stressed, or Depressed Patients

\begin{tabular}{lccc}
\hline Techniques Mentioned When Treating Patients With: & Anxiety & Stress & Depression \\
\hline Pharmacological management considerations - total & $146(85.4 \%)$ & $97(56.7 \%)$ & $29(17.0 \%)$ \\
General medication & $20(11.7 \%)$ & $11(6.4 \%)$ & $3(1.8 \%)$ \\
Oral premedication & $9(5.3 \%)$ & $9(5.3 \%)$ & $4(2.3 \%)$ \\
Sedation & $68(39.8 \%)$ & $48(28.1 \%)$ & $15(8.8 \%)$ \\
Nitrous oxide & $20(11.7 \%)$ & $16(9.4 \%)$ & $2(1.1 \%)$ \\
Postoperative medication & $1(0.6 \%)$ & $1(0.6 \%)$ & $1(0.6 \%)$ \\
Specific medication named & $28(16.4 \%)$ & $12(7.0 \%)$ & $4(2.3 \%)$ \\
Non-pharmacological behavioral management & $107(62.6 \%)$ & $87(50.9 \%)$ & $59(34.5 \%)$ \\
considerations - total & & & \\
General communication & $25(14.6 \%)$ & $19(11.1 \%)$ & $9(5.3 \%)$ \\
Emotion-focused communication & $63(36.8 \%)$ & $53(31.0 \%)$ & $39(22.8 \%)$ \\
Information-focused communication & $19(11.1 \%)$ & $15(8.8 \%)$ & $11(6.4 \%)$ \\
Other management considerations - total & $48(28.1 \%)$ & $55(32.2 \%)$ & $51(29.8 \%)$ \\
Scheduling & $19(11.1 \%)$ & $17(9.9 \%)$ & $12(7.0 \%)$ \\
Referral & $4(2.3 \%)$ & $12(7.0 \%)$ & $23(13.5 \%)$ \\
Other techniques & $25(14.6 \%)$ & $26(15.2 \%)$ & $16(9.4 \%)$ \\
\hline
\end{tabular}


depression. The response category with the highest frequency for patients with depression was the nonpharmacological category $(\mathrm{N}=59 ; 34.5 \%)$. "Sedation" was the most frequently mentioned pharmacological consideration when treating patients with anxiety $(N=68)$, stress $(N=48)$, and depression $(N=15)$.

Concerning the category of non-pharmacological considerations, more periodontists used these techniques when treating patients with anxiety (62.6\%) and stress $(50.9 \%)$ than when treating patients with depression (34.5\%). Of the 107 periodontists (62.6\%) who used special non-pharmacological behavior management techniques when treating anxious patients, the majority ( $\mathrm{N}=63 ; 39.8 \%$ ) used emotion-focused techniques (e.g., "use calm voice," "reassure patient," and "use positive feedback"); fewer respondents $(\mathrm{N}=25 ; 14.6 \%)$ mentioned general communication considerations (e.g., "listening" and "talking") or information-focused communication (e.g., "explanation of procedures" and "answer questions") ( $N=19$; $11.1 \%)$. The same pattern of responses was found concerning the special considerations used when treating patients with high levels of stress (emotionfocused: $31 \%$; general considerations: $11.1 \%$; information-focused: $8.8 \%$ ).

Concerning "other management considerations," one interesting finding was that more providers responded that they would refer patients with depression $(N=23 ; 13.5 \%)$ than patients with anxiety $(2.3 \%)$ or stress $(7.0 \%)$. Other techniques mentioned included offering head phones to listen to music or showing movies.

\section{DISCUSSION}

High levels of anxiety, stress, and depression affect patients' pain perception, their use of pain medication, and wound healing. ${ }^{8-37}$ The connection between the body and mind is a topic of much controversy. However, it seems justified to suggest that psychosocial factors induce the activation of the peripheral sympathetic system and the adrenal medulla. When these stimulations are sustained, they can result in the release of glucocorticoids and mineralocorticoids, which have direct effects on cellular physiology, and lead to reduced wound healing and immune response. ${ }^{38}$ Therefore, considering ways to reduce anxiety, stress, and depression in patients should be part of the treatment considerations. Although this research did not analyze which specific techniques would be the most successful in bringing about a positive change in the patients' psychosocial status, it seems obvious that various pharmacological and non-pharmacological techniques could be used to reach this objective.

This study suggests that providers have a better understanding of the relationships between anxiety and stress and treatment outcomes than the role of depression on their patients' responses to treatment. Providers were less confident in their abilities to detect depression in their patients than in their abilities to detect anxiety and stress. In addition, their repertoire of professional responses when treating patients with high levels of anxiety and stress seems to be richer than when they approach the treatment of patients with depression. Given the ample evidence of the significance of these psychosocial factors on patient response to treatment, it seems important to educate providers about these relationships. Providers should be aware that they can detect depression from nonverbal cues (e.g., body posture and facial expression) and verbal statements (e.g., patients make pessimistic statements that reflect a sense of hopelessness or exhibit a general negativity). In addition, they should be aware that depression has oral implications, such as xerostomia, and it can have an impact on the patient's motivation to engage in good oral hygiene practices and use oral health care services. ${ }^{28-30}$

Based on the responses to the question about whether dental schools had prepared the respondents to consider these matters, dental school curricula should increase coverage of these topics in their education of future health care providers. However, practitioners need to receive more information about these topics as well. Therefore, it seems important to develop continuing education programs and educational materials about these matters that can be accessed easily by busy providers. Kloostra et al. ${ }^{39}$ investigated the relationships among anxiety, stress, and depression and pain perceptions; the use of pain medication; and wound healing following periodontal surgery. Of the three psychosocial factors, depression had the most significant impact on pain perceptions, the use of pain medication, and wound healing. Despite the findings that depression has the greatest impact, the present study showed that depression was the least appreciated psychosocial factor by practicing periodontists. Periodontists reported that they believed that depression had a significantly lower degree of impact on their patients' responses to treatment compared to the roles of anxiety and stress. They believed that depression had the lowest correlation with pain, pain medication use, and wound healing. They were least confident in their abilities to detect it and reported the fewest special techniques used when treating patients with depression. Given the prevalence of depression and the evidence concerning the relationship between depression and treatment outcomes, it seems important to increase efforts to educate periodontists about depression. Therefore, undergraduate and graduate dental school curricula should cover these issues, raise providers' awareness about these matters, and provide them with the knowledge and 
skills needed to respond in the best professional way when treating patients with high levels of anxiety, stress, and depression.

One limitation of this study is the relatively low response rate $(34.41 \%)$, which clearly is at the low end of the acceptability range. ${ }^{40}$ However, one might argue that the periodontists who responded to this self-administered survey were more interested in this topic than the periodontists who did not return the survey. If this assumption is correct, the findings might overestimate the degree to which periodontists are aware of the significance of psychosocial factors when treating their patients.

\section{CONCLUSIONS}

Research showed that anxiety, stress, and depression have a clear relationship with patients' responses to surgical and non-surgical treatment. Although periodontists seem to have a basic understanding of the role of anxiety and stress in their patients' responses to treatment, the ways to detect it in their patients, and how to respond to it, it seems justified to argue that their awareness, knowledge, and skills concerning depression are significantly lower. Therefore, it seems crucial to educate periodontists about the role that psychosocial factors can play in the treatment process, especially the impact of depression on patient response to treatment. Periodontists reported that their dental education had not prepared them well to consider the role of psychosocial factors, such as depression, when treating patients. Educational changes seem necessary.

In addition, given the evidence about how positive coping mechanisms and enhanced perceptions of positive outcomes increase a patient's level of wound healing, ${ }^{11}$ future research should focus on developing targeted interventions that periodontists could use to improve their patients' treatment outcomes, and, thus, their quality of life during treatment.

\section{ACKNOWLEDGMENTS}

This research was supported by funding from the Student Research Program, School of Dentistry, University of Michigan (to PWK). The authors thank the American Academy of Periodontology for making this research possible by giving us access to member addresses. We also thank Dean Peter Polverini and Dr. Laurie McCauley, chair of the Department of Periodontics and Oral Medicine, University of Michigan, for their support of this research.

\section{REFERENCES}

1. Field MJ, ed. Dental Education at the Crossroads Challenges and Change. Washington, DC: National Academy Press; 1995:174-198.
2. Surgeon General's Report on Mental Health. Available at: http://www.surgeongeneral.gov/library/mentalhealth/ home.html. Accessed December 16, 2005.

3. Murray CJ, Lopez AD. Evidence based health policy Lessons from global burden of disease study. Science 1996;274:740-743.

4. Murphy JM, Laird NM, Monson RR, Sobol AM, Leighton AH. A 40 year perspective on the prevalence of depression. Arch Gen Psychiatry 2000;57:209-215.

5. Murphy JM, Horton NJ, Laird NM, Monson RR, Sobol AM, Leighton AH. Anxiety and depression: A 40 year perspective on relationships regarding prevalence, distribution, and comorbidity. Acta Psychiatr Scand 2004;109:355-375.

6. Milgrom P, Weinstein P, Getz T. Treating Fearful Dental Patients: A Patient Management Handbook. Seattle, WA: University of Washington, Continuing Dental Education; 1995.

7. Patel AM, Richards $P$, Wang $H$, Inglehart MR. Surgical or non-surgical periodontal treatment? Factors affecting patient decision making. J Periodontol 2006;77: 678-683.

8. Fox E, O'Boyle C, Barry H, McCreary C. Repressive coping style and anxiety in stressful dental surgery. $\mathrm{Br} J$ Med Psychol 1989;62:371-380.

9. Croog SH, Baume RM, Nalbandian J. Pain response after psychological preparation for repeated periodontal surgery. J Am Dent Assoc 1994;125:1353-1360.

10. Pellino TA, Gordon DB, Engelke ZK, et al. Use of nonpharmacological interventions for pain and anxiety after total hip and total knee arthroplasty. Orthop Nurs 2005;24:182-190, quiz 191-192.

11. Feeney SL. The relationship between pain and negative affect in older adults: Anxiety as a predictor of pain. J Anxiety Disord 2004;18:733-744.

12. Pud D, Amit A. Anxiety as a predictor of pain magnitude following termination of first trimester pregnancy. Pain Med 2005;6:143-148.

13. Holden-Lund C. Effects of relaxation with guided imagery on surgical stress and wound healing. Res Nurs Health 1988;11:235-244.

14. George JM, Scott DS, Turner SP, Gregg JM. The effects of psychological factors and physical trauma on recovery from oral surgery. J Behav Med 1980;3: 291-310.

15. George JM, Scott DS. The effects of psychological factors on recovery from surgery. J Am Dent Assoc 1982;105:251-258.

16. Johannsen A, Asberg M, Soder PO, Soder B. Anxiety, gingival inflammation and periodontal disease in nonsmokers and smokers - An epidemiological study. $J$ Clin Periodontol 2005;32:488-491.

17. Wimmer G, Bratschko RO. Stress, coping, and periodontal disease. In: Mostofsky DI, Forgione AG, Giddon DB, eds. Behavioral Dentistry. Oxford, U.K.: Blackwell/Munksgaard; 2006:139-148.

18. Hilgert JB, Hugo FN, Bandeira DR, Bozzetti MC. Stress, cortisol, and periodontitis in a population aged 50 years and over. J Dent Res 2006;85:324-328.

19. Montgomery GH, Bovbjerg DH. Pre-surgery distress and specific response expectancies predict post surgery outcomes in surgery patients confronting breast cancer. Health Psychol 2004;23:381-387.

20. Genco RJ, Ho AW, Grossi SG, Dunford RG, Tedesco LA. Relationship of stress, distress and inadequate coping behaviors to periodontal disease. J Periodontol 1999;70:711-723. 
21. Wimmer G, Janda M, Wieselmann-Penkner K, Jakse N, Polansky R, Pertl C. Coping with stress: Its influence on periodontal disease. J Periodontol 2002;73:13431351.

22. Rozlog LA, Kiecolt-Glaser JK, Marucha PT, Sheridan JF, Glaser R. Stress and immunity: Implications for viral disease and wound healing. J Periodontol 1999; 70:786-792.

23. Wimmer G, Kohldorfer G, Mischak I, Lorenxoni M, Kallus KW. Coping with stress: Its influence on periodontal therapy. J Periodontol 2005;76:90-98.

24. Takada T, Yoshinari N, Sugiishi S, Kawase H, Yamane $\mathrm{T}$, Noguchi T. Effect of restraint stress on progression of experimental periodontitis in rats. $J$ Periodontol 2004;75:306-315.

25. Giannopoulou C, Kanna JJ, Mombelli A. Effect of inflammation, smoking and stress on gingival crevicular fluid cytokine level. J Clin Periodontol 2003;30: 145-153.

26. Kiecolt-Glaser JK, Marucha PT, Malarkey WB, Mercado AM, Glaser R. Slowing of wound healing by psychological stress. Lancet 1995;346:1194-1196.

27. Spielberger CD. Manual for the State-Trait Anxiety Inventory (Form Y). Palo Alto, CA: Consulting Psychologists Press; 1983.

28. D'Mello DA. Are your patients depressed? Implications for dental practice. J Mich Dent Assoc 2003;85: 26-32.

29. Friedlander AH, Norman DC. Late-life depression: Psychopathology, medical interventions, and dental implications. Oral Surg Oral Med Oral Pathol Oral Radiol Endod 2002;94:404-412.

30. Peeters FP, deVries MW, Vissink A. Risks for oral health with the use of antidepressants. Gen Hosp Psychiatry 1998;20:150-154.

31. Eremenko AG, Vasserman LI, Leosko VA, Li VF. Postoperative pain syndrome and its relationship with individual psychological features of personality (in Russian). Anesteziol I Reanimatol 2001;(4): 24-25.

32. Arpino L, lavarone A, Parlato C, Moraci A. Prognostic role of depression after lumbar disk surgery. Neurol Sci 2004;25:145-147.

33. Field T, Peck M, Krugman S, et al. Burn injuries benefit from massage therapy. J Burn Care Rehabil 1998; 19:241-244.

34. Eggen AE. The Tromso Study: Frequency and predicting factors of analgesic drug use in a free-living population (12-56 years). J Clin Epidemiol 1993;11: 1297-1304.

35. Cole-King A, Harding KG. Psychological factors and delayed healing in chronic wounds. Psychosom Med 2001;63:216-220.

36. Elter JR, White BA, Gaynes BN, Bader JD. Relationship of clinical depression to periodontal treatment outcome. J Periodontol 2002;73:441-449.

37. Monteiro da Silva AM, Oakley DA, Newman HN, Nohl FS, Lloyd HM. Psychosocial factors and adult onset of rapidly progressive periodontitis. J Clin Periodontol 1996;23:789-794.

38. Edward S. Cellular pathophysiology. Part 1: Changes following tissue injury. Prof Nurse 2003;18:562-565.

39. Kloostra PW, Eber R, Wang HL, Inglehart MR. Surgical vs. non-surgical periodontal treatment - Psychosocial factors and treatment outcomes. J Periodontol 2006; 77:1253-1260.

40. Asch DA, Jedrziewski MK, Christakis NA. Response rates to mail surveys published in medical journals. J Clin Epidemiol 1997;50:1129-1136.

Correspondence: Dr. Marita Rohr Inglehart, Department of Periodontics and Oral Medicine, School of Dentistry, University of Michigan, 1011 N. University, Ann Arbor, MI 48109-1078. Fax: 734/ 763-5503; e-mail: mri@umich.edu.

Accepted for publication August 25, 2006. 